\title{
Reasons for not getting vaccinated against COVID-19 in German- speaking Switzerland: An online survey among vaccine hesitant 16- 60 year olds
}

Sara C. Hitchman, Lukas Tribelhorn, Sarah Geber, Thomas N. Friemel

Department of Communication and Media Research, University of Zurich, 15 Andreasstrasse, 8050, Switzerland

Short title: Reasons for not getting COVID-19 vaccine in Switzerland

Keywords: vaccine hesitancy, COVID-19, vaccine confidence, public health, health communication 


\section{Summary}

Background: Several research studies have examined the reasons why people are hesitant to be vaccinated against COVID-19. However, there is no published data to date on Switzerland. Identifying these reasons among the Swiss population who are vaccine hesitant may help inform campaigns to encourage vaccine confidence.

Aims: The primary aim of this study is to identify the reasons for not getting vaccinated against COVID-19 among Swiss residents who are vaccine hesitant. The secondary aim is to examine whether reasons differ by age, gender, education, and likelihood of accepting a vaccination to better target campaigns and design interventions.

Design: An online survey asked participants to indicate the reasons why they were hesitant to be vaccinated against COVID-19.

Setting: German-speaking Swiss Cantons, the survey was administered online between 5 May 2021 and 16 May 2021.

Participants: The participants in this analysis were a sample of $(\mathrm{N}=1191)$ Swiss residents age 16-60 years old from German-speaking Cantons, who could answer an online survey in German, who had yet not been vaccinated, who had not yet registered for a vaccination appointment, and who did not indicate that they would definitely be vaccinated if offered the chance.

Findings: Among people who are vaccine hesitant in Switzerland, the most common reasons for being hesitant were side-effect, safety, and effectiveness concerns. It was also common for people to indicate that they were healthy/at low risk, would decide later, and that they wanted to build immunity naturally. Less common, but still prevalent concerns included wanting more information, thinking COVID-19 was not a real threat, and concerns that the vaccine may serve another purpose. Differences in reasons for being vaccine hesitant were found by age, gender, education, and likelihood of accepting a vaccination if offered.

Conclusions: To increase the likelihood of accepting a vaccination, vaccination campaigns should address side-effect, safety, and effectiveness concerns. Campaigns could also consider informing people why it is necessary for people in lower risk groups to be vaccinated, and why vaccination is preferable to infection for building immunity. While campaigns may be effective in reaching some of the population, alternative strategies might be necessary to strengthen the trust relationship with vaccines and vaccine providers in some groups. Less prevalent concerns, such as not liking needles, could be addressed through individual level interventions. 


\section{Introduction}

COVID-19 vaccination intentions are lower in Switzerland than in other comparable countries [1]. In addition to other public health measures, such as effective contact tracing [2], it will be important to increase vaccination rates, as preventive measures to control the pandemic are relaxed, and the threat of variants of concern continues, for example, the Delta variant [3-5].

Previous research has found that reasons for being hesitant to be vaccinated against COVID-19 in other countries include: concerns around effectiveness and safety, already having had COVID-19, concerns about the newness of vaccine, religious beliefs, lack of trust, not liking needles, not believing that COVID-19 is a risk, a preference for building natural immunity, being against vaccines in general, and belief in conspiracy theories [5-9]. A weekly representative survey of the Swiss population conducted at same time as this study estimated that the number of adults $16-80$ who are undecided or say that it is unlikely that they would accept a vaccination is approximately $19 \%$ (week starting 17 May 2021) [6].

Among people who are vaccine hesitant in Switzerland, this short report examines 1) the reasons that people 16-60 years old in German-speaking Switzerland give for being hesitant to be vaccinated against COVID-19, and 2) differences in the reasons by a) age, b) gender, c) education, and d) likelihood of accepting a vaccination. The focus is on this 'working age' population because these questions were included within an online survey concerned with vaccine hesitancy in younger age groups.

\section{Methods}

\section{Procedures}

Reasons for being vaccine hesitant were asked within an online survey. The online survey asked several questions about COVID-19 generally, COVID-19 vaccination specifically, and additionally tested COVID-19 vaccination campaign messages. This report focuses only on questions about reasons for vaccine hesitancy. Participants were given written information about the study online and indicated consent by clicking yes to participation. The survey lasted approximately 20 minutes and was administered between 5 May 2021 and 16 May 2021. LINK transferred anonymized data to the researchers. Because one portion of the online survey randomised participants to be exposed to three different COVID-19 vaccination poster campaign messages, we checked if the poster message they were exposed to was associated with the reasons they gave. However, there were no significant differences between the groups by the poster they were exposed to (Person's Chi squared test $p<0.05)$. Thus, the poster that they were exposed to was unlikely to have affected the reasons given.

\section{Sample.}

Participants were recruited from the LINK Internet-panel [7]. Briefly, the panel consists of 115,000 members who were recruited actively by LINK in telephone surveys (mobile and landline) including random digit dialling and thereby covering $98 \%$ of the Swiss population. We set age and gender quotas for sampling based on the Swiss population. . A total of $\mathrm{N}=3858$ participants accepted the invitation, after reading study information, $n=43$ did not consent, leading to a total of $n=3815$ to be screened for eligibility. Inclusion and exclusion criteria for the present analysis were: 16-60 years old, 
not vaccinated, not yet registered to be vaccinated, and not willing to be vaccinated in any case if given the chance.

\section{Measures.}

\section{Reasons for not wanting to be vaccinated and vaccination intentions}

Reasons for not wanting to be vaccinated. Participants were asked to indicate the reasons that they were hesitant to be vaccinated against COVID-19. The list contained 17 different reasons based on previous research [8-12]. "Other reason," was also an option, and had an open-text field to write in responses. The order that the reasons appeared in the list were randomised for each participant with the exception of "other" which was always listed last. Participants were instructed to select all reasons that applied. The full list of reasons is listed in English and the original German in Appendix 1.

Likelihood of accepting a COVID-19 vaccination: Participants answered on a 7-point Likert scale (1=under no circumstances vaccinate, $7=$ vaccinate in any case) to the following question: "How likely is it that you would choose to have a COVID-19 vaccination in the coming week if you had the chance?" The variable was recoded into three levels of likelihood of accepting a vaccination: very unlikely (scores 1 and 2), rather unlikely (scores 3 and 4), and likely (scores 5 and 6). Participants indicating 7 (would vaccinate in any case) were not asked about reasons for not vaccinating and were excluded.

\section{Demographic characteristics}

Gender. Participants were asked, "Are you, female, male, or other?" Participants who selected other could write in an answer. One participant who wrote in "other" was removed from the analyses on gender differences but was included in all other analyses.

Age. Participants were asked, "What is your age?" Participants entered their age numerically. Age was grouped as: $16-30,31-45$, and $46-60$.

Education. Participants were asked, "What is the highest education level that you have completed with a certificate or diploma?" Response options included: 1) no compulsory school, 2) compulsory school, 3) "Haushaltslehrjahr," or "Handelsschule", 4) (short) Apprenticeship, 5) Diploma school or secondary technical school, 6) Vocational apprenticeship, 7) Vocational schools, 8) Upper secondary schools, or 9) University or tertiary education. There is no common international equivalent of "Haushaltslehrjahr" or "Handelsschule," this schooling involves basic training in running a home or business. These were grouped as: 1-3 = Low; 4-8 = Medium; $9=$ High to understand participant characteristics. For analyses purposes, the low and medium groups were combined due to small numbers in the low education group.

\section{Analyses}

All analyses were conducted in $R$ version 4.0.4. Sample sizes and percentages presented are unweighted. Unweighted Pearson's Chi-squared Tests were used to test differences in the reasons supported by age, gender, education, and vaccine confidence, with Yates' continuity correction for the education and gender analyses (binary variables). We ran several Chi-squared tests leading to the possibility that some of the differences found may be due to multiple testing (chance findings). A more 
cautious interpretation could adjust the $p$-value to conclude that differences may exist, e.g., $p<0.003$ using the Bonferroni correction for each Table of results.

The responses of the participants who provided "other" reasons for not getting vaccinated were analysed qualitatively. Responses were first read over by the researchers. A simple coding frame of reasons was generated inductively. Responses were then iteratively sorted into the coding frame. These codes were then sorted into broader factors to allow them to be summarised.

\section{Results}

\section{Participants}

The study focuses on people who are vaccine hesitant in Switzerland. This sample was arrived at as follows. Of the $n=3815$ participants who gave consent to participate, $n=67$ were over the age of 60 and were excluded, $n=3748$ went on to indicate vaccination status, $n=1459$ were excluded because they had already received at least one vaccine dose, leaving a sample of $n=2289$. For this analysis, we further excluded $n=1022$ participants who had already registered to be vaccinated, but had not yet been vaccinated, leaving $n=1267$. Reasons for being vaccine hesitant were then only asked among people who did not say they would vaccinate in any case, e.g., scores 1-6 on likelihood of accepting a vaccination, excluding scores of $7(n=76)$ participants, leaving a final sample size of $n=1191$. All participants gave an answer to each of the relevant questions, thus there was no missing data.

Table 1 shows the characteristics of the current study participants, the initial sample characteristics from which the sample was drawn $(\mathrm{N}=3748)$, and the prevalence of the characteristics in the Swiss population. Most participants of the current study were 46-60, female, had a medium level of education, and tended towards a low likelihood of accepting a vaccination. Note that the sample characteristics reflect the inclusion and exclusion criteria (e.g., participants not yet registered to be vaccinated, and who would not vaccinate in any case), and the initial study sample ( $N=3748)$ from which the sample for the current study was drawn $(\mathrm{N}=1191)$. Compared to the general Swiss population females, and older age groups were overrepresented in the initial sample. Therefore, the characteristics of the analysed sample should not be used as a basis for inferences on the sociodemographic characteristics of people who are vaccine hesitant.

[Table 1 here]

\section{Main findings}

The number and percent of participants who indicated each reason for being hesitant to get vaccinated against COVID-19 are shown in Table 2. The most common reasons for not vaccinating were side-effect, safety, and effectiveness concerns. Belief that they were healthy and not at risk, wanting to build immunity naturally, and saying they would decide later were also common reasons. Less prevalent concerns included, not trusting authorities, believing COVID-19 was not a real threat, believing the vaccines served another purpose, wanting more information, and being against vaccination in general. Fewer participants indicated that they had already had COVID-19, that they did not like needles/injections, and that the vaccine was not compatible with their health conditions. Very few participants indicated not having enough time or religious concerns.

[Table 2 here] 
Age differences in reasons for not getting vaccinated against COVID-19. See Table 3 for results. A higher percentage of older participants (46-60 years old) indicated that they did not want to get vaccinated against COVID-19 because they had safety concerns about the vaccine, had health conditions it was not compatible with, wanted to build immunity naturally, were against vaccination generally, were concerned the vaccines served another purpose, and did not trust the authorities advising it. A higher percentage of younger participants (16-30) reported not wanting to get vaccinated because they needed more information, and because they did not like needles/injections.

[Table 3 here]

Gender differences in reasons for not getting vaccinated against COVID-19. See Table 4 for results. A higher percentage of males than females reported that they did not want to get vaccinated because they believed that COVID-19 was not a real threat, did not like needles/injections, believed enough other people would get vaccinated, and did not trust the authorities advising it. A higher percentage of females than males reported not wanting to be vaccinated because they had concerns about vaccine effectiveness.

[Table 4 here]

Education differences in reasons for not getting vaccinated against COVID-19. See Table 5 for results. A higher percentage of participants with low/moderate education than high education reported that they did not want to get vaccinated because they had concerns about side-effects, believed that enough other people would get vaccinated, and had concerns that the vaccine serves another purpose.

[Table 5 here]

Likelihood of accepting a vaccination differences in reasons for not getting vaccinated against COVID19. See Table 6 for results. There were differences in reasons for not getting vaccinated by likelihood of accepting a vaccination for every reason except for already having had COVID-19 and not liking needles/injections. Briefly, among those who are very unlikely to accept a vaccine, safety, side-effect, and effectiveness concerns were very high along with believing they were healthy and at low risk, wanting to build immunity naturally, not trusting the authorities advising it, having concerns that the vaccines serve another purpose, and being against vaccination generally. Those rather unlikely to accept a vaccine more often reported needing more information, and saying they would decide later. Participants likely to accept a vaccination still had concerns (e.g., safety, side-effect, and effectiveness), but overall a lower percentage endorsed each reason for not vaccinating.

[Table 6 here]

Other reasons for not vaccinating. Based on the analysis of the open text responses, we grouped the other reasons given into five factors: 1) lack of long-term research, 2) effectiveness concerns, 3) mistrust, rumours and misperceptions about vaccines, 4) belief that they are healthy and risk is low, and 5) pre-existing conditions, and practical factors. 1) Lack of long-term research: The other reasons that participants wrote in for not vaccinating overwhelmingly included concerns about the long-term effects of vaccination and the speed of vaccine development. Of the participants indicating these reasons, several expressed explicit concerns about the newness of mRNA vaccines, with a few saying they would get vaccinated with a non-mRNA vaccine. 2) Effectiveness concerns: various effectiveness concerns were common, there was confusion over whether getting vaccinated would prevent them from transmitting COVID-19 to others, worries that the vaccine would only offer 
protection for a short period of time, that mutations would lead to changes in effectiveness, and that unwanted annual vaccinations would be necessary. 3) Mistrust, rumours and misperceptions about vaccines: Several participants gave reasons that expressed a lack of trust in vaccines, and a belief in rumours and misperceptions, such that the vaccines were only to make money, that they could cause infertility, that breastfeeding prevented vaccination [13], and that COVID-19 was a hoax. 4) Belief that they are healthy and not at risk: Several participants felt that they were not personally at risk, and that their immune system was strong enough. 5) Pre-existing conditions and practical factors: Allergies, auto-immune disorders and pregnancy were given as reasons for not vaccinating, along with trouble booking an appointment by a few participants. It should be noted that the option for pregnant women to be vaccinated under certain conditions was introduced in Switzerland shortly after this survey was conducted [14].

\section{Discussion}

Health behaviour theories describe how health protective behaviours can be motivated by perceived risk of the protective behaviour and the threat itself, and the effectiveness of the behaviour in protecting against the risk [15]. In line with these behavioural theories, the results show the most common reasons for being vaccine hesitant are the perceived risks of the vaccination, including sideeffects and safety concerns. This finding is similar to previous research on vaccine confidence in Switzerland that suggests there are relatively high concerns about vaccine safety [16]. Vaccine effectiveness was an additional common concern, which aligns with behavioural theories as well.

Low perceived risks of COVID-19 were also common reasons for being vaccine hesitant (e.g., feeling at low risk, wanting to build immunity naturally, and thinking that COVID-19 is not a real threat). Not trusting the authorities advising the vaccine and concern the vaccines may serve another purpose, although less common, were further prevalent reasons. In addition to alignment with health behaviour theories, these findings align with research on the "trust relationship" with vaccines, where trust of the product (vaccine), trust in the provider (health professional), and the trust of the political/system (authority) are important components of the vaccine decision making process [17].

There were several differences in the reasons supported by age, gender, education, and likelihood of accepting a vaccination, we discuss the most notable differences here. Age differences included a higher number of participants in older age groups who endorsed reasons such as wanting to build immunity naturally, and the higher number of young people wanting more information. Age differences may be due to older groups having been eligible longer for the vaccines, and older people not yet vaccinated or registered to be vaccinated likely have lower vaccine confidence than younger groups.

For gender, there was a higher number of males who said enough other people would get vaccinated. Males were also more likely to endorse that COVID-19 was not a real threat, and that they did not like needles/injections. There were few differences by education, notably lower education groups were more likely to endorse the concern that the vaccines may serve another purpose as a reason.

Not surprisingly, the largest differences in the reasons given were by likelihood of accepting a vaccination. For example, in our analyses participants with the lowest likelihood of accepting a vaccine more often reported wanting to build immunity naturally, not trusting the authorities who advised the vaccine, and believing COVID-19 was not a real threat, alongside their concerns about vaccine safety, side-effects, and effectiveness. For participants who were rather unlikely to accept a vaccine, alongside their concerns about vaccine safety, side-effects, and effectiveness, a higher number than other groups said they would decide later, and that they wanted more information. For participants with the highest likelihood of accepting a vaccine among our vaccine hesitant sample, concerns about vaccine safety, side-effects, and effectiveness remained, with high numbers also reporting that they were healthy and not at risk and would decide later. 
Several reasons for not vaccinating were identified in the "other" open-text responses. Reasons that differed from the ones we asked about in the survey included specific concerns about the long-term effects of the vaccines, and speed at which the vaccines were developed. There was also concern and confusion around how long the vaccines would be effective for, whether this would change due to mutants, and the necessity of annual vaccination. Overall, the reasons written in the other responses mirrored the major concerns we found in the quantitative data, such that side-effects, safety, and effectiveness concerns were the most common.

Limitations: The study was only conducted among the Swiss-German speaking regions, and only among 16-60 year olds. While this may limit generalisability to other areas of Switzerland and older people, this is still an important target group. When restrictions lift, the population $16-60$ years old, many whom will be working or attending education, will begin moving and interacting with others more, potentially spreading the virus. Another limitation is that this study was conducted within a study testing three different poster campaign messages. However, we found no difference in the reasons for vaccine hesitancy by the poster campaign message that participants were exposed to. Additionally, one of the posters (control condition) was similar to messages in the current live campaign in Switzerland. Thus, most people would have already seen a similar message to the control.

\section{Conclusions}

We have identified key reasons for not getting vaccinated amongst people in Switzerland who remain hesitant to be vaccinated against COVID-19. These reasons differ by, age, gender, education, and likelihood of accepting a vaccination. Future vaccination media campaigns and interventions will need to take into consideration that people who remain vaccine hesitant in Switzerland have concerns that will likely need to be addressed by building trust in COVID-19 vaccines, and increasing the belief that COVID-19 is a serious threat. Younger age groups, who currently are less likely to report not trusting authorities and related reasons, may benefit from targeted information and prompts to get vaccinated. 


\section{References}

1 Lacey N. COVID-19 vaccination intent has soared across the world. www.ipsos.com 2021, Mar 11 [updated 2021 Mar 11; cited 2021 May 26]. Available from: https://www.ipsos.com/en/covid-19vaccination-intent-has-soared-across-world.

2 Salathé M, Althaus CL, Neher R, Stringhini S, Hodcroft E, Fellay J et al. COVID-19 epidemic in Switzerland: on the importance of testing, contact tracing and isolation. Swiss Medical Weekly 2020;150(1112):w20225. 10.4414/smw.2020.20225.

3 Abdool Karim SS, Oliveira T de. New SARS-CoV-2 Variants - Clinical, Public Health, and Vaccine Implications. The New England journal of medicine 2021;384(19):1866-8. 10.1056/NEJMc2100362.

4 Public Health England. Risk assessment for SARS-CoV-2 variant: VOC-21APR-02 (B.1.617.2) 2021.

5 Q\&A: COVID-19 variants and what they mean for countries and individuals: World Health Organization; 2021 [updated 2021 May 21; cited 2021 May 27]. Available from: https://www.euro.who.int/en/health-topics/health-emergencies/coronavirus-covid19/news/news/2021/5/q-and-a-covid-19-variants-and-what-they-mean-for-countries-andindividuals.

6 Covid-Norms | Monitoring and Analyzing Preventive Behavior; 2021 [updated 2021 May 27; cited 2021 May 27]. Available from: https://covid-norms.ch/en/.

7 LINK. Products: LINK Panel | LINK; 2021 [updated 2021 Feb 8; cited 2021 Jun 21]. Available from: https://www.link.ch/en/products/the-link-internet-panel/.

8 Al-Qerem WA, Jarab AS. COVID-19 Vaccination Acceptance and Its Associated Factors Among a Middle Eastern Population. Frontiers in public health 2021;9:632914. 10.3389/fpubh.2021.632914.

9 Callaghan T, Moghtaderi A, Lueck JA, Hotez P, Strych U, Dor A et al. Correlates and disparities of intention to vaccinate against COVID-19. Social science \& medicine (1982) 2021;272:113638. 10.1016/j.socscimed.2020.113638.

10 Fisher KA, Bloomstone SJ, Walder J, Crawford S, Fouayzi H, Mazor KM. Attitudes Toward a Potential SARS-CoV-2 Vaccine A Survey of U.S. Adults. Annals of internal medicine 2020;173(12):964-73. 10.7326/M20-3569.

11 Ruiz JB, Bell RA. Predictors of intention to vaccinate against COVID-19: Results of a nationwide survey. Vaccine 2021;39(7):1080-6. 10.1016/j.vaccine.2021.01.010.

12 Yoda T, Katsuyama H. Willingness to Receive COVID-19 Vaccination in Japan. Vaccines 2021;9(1). 10.3390/vaccines9010048. 
13 Promotion allaitement maternel Suisse. Vaccination COVID-19 dans la grossesse; 2021 [updated 2021 Jun 10; cited 2021 Jun 10]. Available from:

https://www.stillfoerderung.ch/logicio/pmws/stillen_corona_fr.html.

14 GYNÉCOLOGIE SUISSE SGGG. Communiqué de presse de SSGO gynécologie suisse du 28 mai 2021: Extension de la recommandation vaccinale des femmes enceintes contre le COVID-19 [2021 Jun 21]. Available from:

https://www.sggg.ch/fileadmin/user_upload/Communique_de_presse_de_SSGO_gynecologie_sui sse_du_28_mai_2021.pdf.

15 Glanz K, Bishop DB. The role of behavioral science theory in development and implementation of public health interventions. Annual review of public health 2010;31:399-418. 10.1146/annurev.publhealth.012809.103604.

16 Wellcome. Chapter 5: Attitudes to vaccines | Wellcome Global Monitor 2018; 2021 [updated 2021 May 27; cited 2021 May 27]. Available from: https://wellcome.org/reports/wellcome-globalmonitor/2018/chapter-5-attitudes-vaccines.

17 Larson HJ, Clarke RM, Jarrett C, Eckersberger E, Levine Z, Schulz WS et al. Measuring trust in vaccination: A systematic review. Human Vaccines \& Immunotherapeutics 2018;14(7):1599-609. 10.1080/21645515.2018.1459252. 
Appendix 1 - Reasons against vaccination, German version from survey, and English translation

Original

Was sind die Gründe, die für Sie gegen eine Covid-19 Impfung sprechen?

Bitte wählen Sie alles aus, was zutrifft.

1 Ich hatte bereits Covid-19

2 Ich habe Bedenken bezüglich der Sicherheit des Impfstoffs

3 Ich habe Bedenken bezüglich der Wirksamkeit des Impfstoffs

4 Ich habe Bedenken wegen der Nebenwirkungen des Impfstoffs
Translation

What are the reasons that speak against a

Covid-19 vaccination for you?

Please select everything that applies.

1 I already had Covid-19

2 I have concerns about the safety of the vaccine

3 I have concerns about the effectiveness of the vaccine

4 I have concerns about vaccine sideeffects

5 Der Impfstoff ist mit meinem Gesundheitszustand nicht $\mathbf{5}$ The vaccine is not compatible with my vereinbar (z.B. Allergien) health conditions (e.g., allergies)

6 Ich bin gesund und habe ein geringes Risiko für Covid- $6 \mathrm{I}$ am healthy and at low risk for Covid-19 19

7 Die Impfung ist unnötig, da Covid-19 keine echte Bedrohung darstellt

8 Ich würde lieber eine Immunität durch eine Infektion aufbauen als durch eine Impfung

9 Meine religiösen Überzeugungen

10 Ich bin generell gegen eine Impfung

11 Ich mag keine Nadeln/Injektionen

12 Ich brauche mehr Informationen

13 Ich habe nicht genug Zeit, um mich impfen zu lassen

14 Es ist nicht nötig, dass ich mich impfen lasse, da es genug andere gibt, die gegen Covid-19 geimpft werden

15 Bedenken, dass die Impfstoffe einen anderen Zweck haben

16 Ich werde mich später entscheiden

17 Ich traue den Behörden nicht, die den Impfstoff empfehlen

18 Andere, nämlich
7 The vaccination is unnecessary as Covid19 is not a real threat

8 I would rather build immunity through infection than vaccination

9 My religious beliefs

$10 \mathrm{I}$ am against vaccination in general

11 I do not like needles/injections

12 I need more information

13 I do not have enough time to get vaccinated

14 There is no need that I get vaccinated as there will enough others who will be vaccinated against Covid-19

15 Concern the vaccines have another purpose

16 I will decide later

17 I do not trust authorities who recommend the vaccine

18 Other, namely

Answer options were binary multiple choice (yes/no). Items were randomized while 18 was always last. 
Declaration of competing interests: None to declare.

Ethics committee approval: This study has received ethical approval from Ethics Committee of the Faculty of Arts and Social Sciences of the University of Zurich (approval number 21.4.9).

Financial support: This study was funded by the Swiss National Science Foundation NFP 78, 4078P0_198299 / 1 - Covid-Norms.

Correspondence to Thomas N. Friemel, Department of Communication and Media Research, University of Zurich, 15 Andreasstrasse, 8050, Zurich, Switzerland. E-mail: th.friemel@ikmz.uzh.ch 
Table 1. Sample Characteristics

\begin{tabular}{lccccc}
\hline \multirow{2}{*}{ Characteristic } & $\begin{array}{c}\text { Current study } \\
\text { sample, } \\
\text { N=1191 }\end{array}$ & $\begin{array}{c}\text { Original sample, } \\
\mathbf{N = 3 7 4 8}\end{array}$ & $\begin{array}{c}\text { Swiss } \\
\text { Population }\end{array}$ \\
\cline { 2 - 6 } & $\mathbf{n}$ & $\mathbf{\%}$ & $\mathrm{n}$ & $\%$ & $\%$ \\
\hline Age & 253 & $21 \%$ & 532 & $14 \%$ & $29 \%$ \\
$\quad 16-30$ & 426 & $36 \%$ & 1213 & $32 \%$ & $35 \%$ \\
$31-45$ & 512 & $43 \%$ & 2003 & $53 \%$ & $36 \%$ \\
$\quad 46-60$ & & & & & \\
Gender & 512 & $43 \%$ & 1737 & $46 \%$ & $51 \%$ \\
$\quad$ Male & 678 & $57 \%$ & 2004 & $54 \%$ & $49 \%$ \\
$\quad$ Female & 1 & $0 \%$ & 7 & $0,20 \%$ & \\
$\quad$ Other & & & & & \\
Education & 50 & $4 \%$ & 113 & $3 \%$ & \\
$\quad$ Low & 756 & $63 \%$ & 2121 & $57 \%$ & \\
$\quad \begin{array}{l}\text { Medium } \\
\text { High }\end{array}$ & 385 & $32 \%$ & 1514 & $40 \%$ & \\
Likelihood of vaccination & & & & & \\
$\quad$ Unlikely & 644 & $54 \%$ & & & \\
$\quad$ Rather unlikely & 331 & $28 \%$ & & & \\
$\quad$ Likely & 216 & $18 \%$ & & & \\
\hline \hline
\end{tabular}


Table 2. Reasons for not getting vaccinated against COVID-19, N=1191

\begin{tabular}{lcc} 
Reasons for not vaccinating & $\begin{array}{c}\text { n support } \\
\text { reason }\end{array}$ & $\begin{array}{c}\text { \% } \\
\text { support } \\
\text { reason }\end{array}$ \\
\hline Side-effects concerns & 760 & $64 \%$ \\
Safety concerns & 735 & $62 \%$ \\
Effectiveness concerns & 641 & $54 \%$ \\
Healthy, at low-risk & 494 & $41 \%$ \\
Will decide later & 440 & $37 \%$ \\
Want to build immunity naturally & 408 & $34 \%$ \\
Do not trust the authorities who advise it & 316 & $27 \%$ \\
Need more information & 268 & $23 \%$ \\
Concern the vaccines have another purpose & 248 & $21 \%$ \\
COVID-19 not a real threat & 239 & $20 \%$ \\
Against vaccination in general & 222 & $19 \%$ \\
Do not like needles/injections & 178 & $15 \%$ \\
Already had COVID-19 & 146 & $12 \%$ \\
Enough other people will get vaccinated & 105 & $9 \%$ \\
Not compatible with health conditions (e.g., allergies) & 102 & $8 \%$ \\
Not enough time & 22 & $2 \%$ \\
Religion & 18 & $2 \%$ \\
Other reason & 183 & $15 \%$ \\
\hline \hline
\end{tabular}


Table 3. Reasons for not getting vaccinated against COVID-19 by age group, $N=1191$

\begin{tabular}{|c|c|c|c|c|c|c|c|}
\hline \multirow[b]{2}{*}{ Reasons for not vaccinating } & \multicolumn{2}{|c|}{$16-30$} & \multicolumn{2}{|c|}{$31-45$} & \multicolumn{2}{|c|}{$46-60$} & \multirow{2}{*}{$\begin{array}{l}\text { Chi- } \\
\text { squared } p \text { - } \\
\text { valuet }\end{array}$} \\
\hline & $\mathbf{n}$ & $\%$ & $\mathbf{n}$ & $\%$ & $\mathbf{n}$ & $\%$ & \\
\hline Side effects concerns & 151 & $60 \%$ & 265 & $62 \%$ & 344 & $67 \%$ & 0,0877 \\
\hline Safety concerns & 137 & $54 \%$ & 257 & $60 \%$ & 341 & $67 \%$ & 0,0030 \\
\hline Effectiveness concerns & 124 & $49 \%$ & 226 & $53 \%$ & 291 & $57 \%$ & 0,1148 \\
\hline Healthy, at low-risk & 103 & $41 \%$ & 187 & $44 \%$ & 204 & $40 \%$ & 0,4380 \\
\hline Will decide later & 97 & $38 \%$ & 155 & $36 \%$ & 188 & $37 \%$ & 0,8694 \\
\hline Need more information & 73 & $29 \%$ & 107 & $25 \%$ & 88 & $17 \%$ & 0,0004 \\
\hline Want to build immunity naturally & 62 & $25 \%$ & 143 & $34 \%$ & 203 & $40 \%$ & 0,0002 \\
\hline Do not trust the authorities who advise it & 57 & $23 \%$ & 99 & $23 \%$ & 160 & $31 \%$ & 0,0058 \\
\hline Do not like needles/injections & 48 & $19 \%$ & 71 & $17 \%$ & 59 & $12 \%$ & 0,0115 \\
\hline COVID-19 not a real threat & 44 & $17 \%$ & 82 & $19 \%$ & 113 & $22 \%$ & 0,2742 \\
\hline Against vaccination in general & 38 & $15 \%$ & 70 & $16 \%$ & 114 & $22 \%$ & 0,0184 \\
\hline Concern the vaccines have another purpose & 39 & $15 \%$ & 87 & $20 \%$ & 122 & $24 \%$ & 0,0256 \\
\hline Already had COVID-19 & 30 & $12 \%$ & 38 & $9 \%$ & 78 & $15 \%$ & 0,0131 \\
\hline Enough other people will get vaccinated & 25 & $10 \%$ & 41 & $10 \%$ & 39 & $8 \%$ & 0,4451 \\
\hline Not compatible with health conditions & 11 & $4 \%$ & 36 & $8 \%$ & 55 & $11 \%$ & 0,0120 \\
\hline Not enough time* & 10 & $4 \%$ & 5 & $1 \%$ & 7 & $1 \%$ & 0,0192 \\
\hline Religion* & 3 & $1 \%$ & 5 & $1 \%$ & 10 & $2 \%$ & 0,5550 \\
\hline
\end{tabular}

*interpreted with caution due to low cell counts

tbold indicates significant at $p<0.05$ 
Table 4. Reasons for not getting vaccinated against COVID-19 by gender, $N=1190$

\begin{tabular}{|c|c|c|c|c|c|}
\hline \multirow[b]{2}{*}{ Reasons for not vaccinating } & \multicolumn{2}{|c|}{ Male } & \multicolumn{2}{|c|}{ Female } & \multirow{2}{*}{$\begin{array}{c}\text { Chi- } \\
\text { squared } \\
\text { p-value* }\end{array}$} \\
\hline & $\mathbf{n}$ & $\%$ & $\mathbf{n}$ & $\%$ & \\
\hline Side effects concerns & 315 & $62 \%$ & 444 & $65 \%$ & 0,1778 \\
\hline Safety concerns & 309 & $60 \%$ & 425 & $63 \%$ & 0,4477 \\
\hline Effectiveness concerns & 258 & $50 \%$ & 382 & $56 \%$ & 0,0477 \\
\hline Healthy, at low-risk & 228 & $45 \%$ & 265 & $39 \%$ & 0,0674 \\
\hline Will decide later & 187 & $37 \%$ & 253 & $37 \%$ & 0,8262 \\
\hline Want to build immunity naturally & 180 & $35 \%$ & 227 & $33 \%$ & 0,5882 \\
\hline Do not trust the authorities who advise it & 158 & $31 \%$ & 157 & $23 \%$ & 0,0035 \\
\hline COVID-19 not a real threat & 129 & $25 \%$ & 110 & $16 \%$ & 0,0002 \\
\hline Concern the vaccines have another purpose & 117 & $23 \%$ & 130 & $19 \%$ & 0,1398 \\
\hline Need more information & 104 & $20 \%$ & 164 & $24 \%$ & 0,1298 \\
\hline Against vaccination in general & 98 & $19 \%$ & 124 & $18 \%$ & 0,7656 \\
\hline Do not like needles/injections & 95 & $19 \%$ & 83 & $12 \%$ & 0,0033 \\
\hline Enough other people will get vaccinated & 66 & $13 \%$ & 38 & $6 \%$ & $<0,0001$ \\
\hline Already had COVID-19 & 61 & $12 \%$ & 84 & $12 \%$ & 0,8739 \\
\hline Not compatible with health conditions & 34 & $7 \%$ & 67 & $10 \%$ & 0,0599 \\
\hline Religion & 9 & $2 \%$ & 9 & $1 \%$ & 0,7171 \\
\hline Not enough time & 11 & $2 \%$ & 11 & $2 \%$ & 0,653 \\
\hline
\end{tabular}

*bold indicates significant at $p<0.05$ 
Table 5. Reasons for not getting vaccinated against COVID-19 by education level, $N=1191$

\begin{tabular}{lccccc}
\hline \multicolumn{1}{c}{ Reasons for not vaccinating } & $\begin{array}{c}\text { Low/Medium } \\
\text { Education }\end{array}$ & $\begin{array}{c}\text { High } \\
\text { Education }\end{array}$ & $\begin{array}{c}\text { Chi- } \\
\text { square p- } \\
\text { value* }\end{array}$ \\
\cline { 2 - 4 } Side effects concerns & $\mathbf{n}$ & $\mathbf{\%}$ & $\mathbf{n}$ & $\mathbf{\%}$ & $\mathbf{0 , 0 1 9 1}$ \\
Safety concerns & 533 & $66 \%$ & 227 & $59 \%$ & $\mathbf{0 , 0}$ \\
Effectiveness concerns & 509 & $63 \%$ & 226 & $59 \%$ & 0,1574 \\
Healthy, at low-risk & 450 & $56 \%$ & 191 & $50 \%$ & $\mathbf{0 , 0 5 0 9}$ \\
Will decide later & 322 & $40 \%$ & 172 & $45 \%$ & 0,1375 \\
Want to build immunity naturally & 298 & $37 \%$ & 142 & $37 \%$ & 1,0000 \\
Do not trust the authorities who advise it & 276 & $34 \%$ & 132 & $34 \%$ & 1,0000 \\
Concern the vaccines have another purpose & 223 & $28 \%$ & 93 & $24 \%$ & 0,2249 \\
Need more information & 186 & $23 \%$ & 62 & $16 \%$ & $\mathbf{0 , 0 0 7}$ \\
COVID-19 not a real threat & 178 & $22 \%$ & 90 & $23 \%$ & 0,6706 \\
Against vaccination in general & 169 & $21 \%$ & 70 & $18 \%$ & 0,2958 \\
Do not like needles/injections & 154 & $19 \%$ & 68 & $18 \%$ & 0,6037 \\
Already had COVID-19 & 124 & $15 \%$ & 54 & $14 \%$ & 0,5973 \\
Enough other people will get vaccinated & 89 & $11 \%$ & 57 & $15 \%$ & 0,0788 \\
Not compatible with health conditions & 81 & $10 \%$ & 24 & $6 \%$ & $\mathbf{0 , 0 3 9 1}$ \\
Not enough time & 73 & $9 \%$ & 29 & $8 \%$ & 0,4421 \\
Religion & 13 & $2 \%$ & 9 & $2 \%$ & 0,5230 \\
\hline \hline
\end{tabular}

*bold indicates significant at $p<0.05$ 
Table 6. Reasons for not getting vaccinated against COVID-19 by likelihood of accepting a vaccination, N=1191

\begin{tabular}{|c|c|c|c|c|c|c|c|}
\hline \multirow{3}{*}{ Reasons for not vaccinating } & \multicolumn{6}{|c|}{ Likelihood of accepting a vaccination } & \multirow{3}{*}{$\begin{array}{c}\text { Chi- } \\
\text { squared } \\
\text { p- } \\
\text { valuet }^{-}\end{array}$} \\
\hline & \multicolumn{2}{|c|}{ Unlikely } & \multicolumn{2}{|c|}{$\begin{array}{l}\text { Rather } \\
\text { Unlikely }\end{array}$} & \multicolumn{2}{|c|}{ Likely } & \\
\hline & $\mathbf{n}$ & $\%$ & $\mathrm{n}$ & $\%$ & n & $\%$ & \\
\hline Safety concerns & 457 & $71 \%$ & 183 & $55 \%$ & 95 & $44 \%$ & $<0,0001$ \\
\hline Side effects concerns & 440 & $68 \%$ & 208 & $63 \%$ & 112 & $52 \%$ & $<0,0001$ \\
\hline Effectiveness concerns & 402 & $62 \%$ & 159 & $48 \%$ & 80 & $37 \%$ & $<0,0001$ \\
\hline Healthy, at low-risk & 326 & $51 \%$ & 105 & $32 \%$ & 63 & $29 \%$ & $<0,0001$ \\
\hline Want to build immunity naturally & 300 & $47 \%$ & 72 & $22 \%$ & 36 & $17 \%$ & $<0,0001$ \\
\hline Do not trust the authorities who advise it & 257 & $40 \%$ & 46 & $14 \%$ & 13 & $6 \%$ & $<0,0001$ \\
\hline COVID-19 not a real threat & 211 & $33 \%$ & 21 & $6 \%$ & 7 & $3 \%$ & $<0,0001$ \\
\hline Concern the vaccines have another purpose & 205 & $32 \%$ & 32 & $10 \%$ & 11 & $5 \%$ & $<0,0001$ \\
\hline Against vaccination in general & 180 & $28 \%$ & 36 & $11 \%$ & 6 & $3 \%$ & $<0,0001$ \\
\hline Will decide later & 174 & $27 \%$ & 187 & $56 \%$ & 79 & $37 \%$ & $<0,0001$ \\
\hline Need more information & 138 & $21 \%$ & 93 & $28 \%$ & 37 & $17 \%$ & 0,0069 \\
\hline Do not like needles/injections & 93 & $14 \%$ & 51 & $15 \%$ & 34 & $16 \%$ & 0,8641 \\
\hline Already had COVID-19 & 86 & $13 \%$ & 35 & $11 \%$ & 25 & $12 \%$ & 0,4304 \\
\hline Enough other people will get vaccinated & 83 & $13 \%$ & 17 & $5 \%$ & 5 & $2 \%$ & $<0,0001$ \\
\hline Not compatible with health conditions & 74 & $11 \%$ & 21 & $6 \%$ & 7 & $3 \%$ & 0,0002 \\
\hline Religion* & 16 & $2 \%$ & 1 & $1 \%$ & 1 & $1 \%$ & 0,0114 \\
\hline Not enough time* & 4 & $1 \%$ & 11 & $3 \%$ & 7 & $3 \%$ & 0,0030 \\
\hline
\end{tabular}

*interpreted with caution due to small cell counts

tbold indicates significant at $p<0.05$ 